\title{
Modélisation des documents numériques pour l'histoire des techniques : une perspective de recherche
}

Modelisation of digital documents for history of technology : a perspective of research

Sylvain Laubé

\section{OpenEdition Journals}

Édition électronique

URL : http://journals.openedition.org/dht/160

DOI : $10.4000 /$ dht. 160

ISSN : $1775-4194$

Éditeur :

Centre d'histoire des techniques et de l'environnement du Cnam (CDHTE-Cnam), Société des élèves du CDHTE-Cnam

\section{Édition imprimée}

Date de publication : 1 décembre 2009

Pagination : $37-41$

ISBN : 978-2-9530779-4-0

ISSN : 0417-8726

Référence électronique

Sylvain Laubé, « Modélisation des documents numériques pour l'histoire des techniques : une perspective de recherche », Documents pour l'histoire des techniques [En ligne], $1812^{\mathrm{e}}$ semestre 2009, mis en ligne le 28 décembre 2010, consulté le 08 septembre 2020. URL : http:// journals.openedition.org/dht/160 ; DOI : https://doi.org/10.4000/dht.160 


\title{
Modélisation des documents numériques pour l'histoire des techniques: une perspective de recherche
}

\author{
Sylvain Laubé \\ Équipe « Patrimoine, histoire des sciences et techniques " (PaHST) \\ Université de Bretagne Occidentale
}

\begin{abstract}
RÉsumé
Partant de travaux de recherches menés à Brest dans les trois domaines suivants : I'histoire des techniques du port-arsenal de Brest, l'éducation et l'ingénierie des environnements informatiques pour l'apprentissage humain (EIAH), cette note aura pour objet d'expliciter les problèmes posés aux historiens par l'usage des $\mathrm{TIC}$ et en quoi cela se traduit par un nouvel axe de recherche dans l'équipe PaHST à l'Université de Brest. À partir du constat que l'on trouve aisément sur le web des sites dits « historiques » qui publient des images de synthèse, des animations 3D cinématiques, (et au travers de l'exemple du pont tournant de Brest construit dans la seconde moitié du XIXe siècle), deuX aspects seront discutés : 1) I'historicité de ces images (qui implique une explicitation de la méthode historique à l'œuvre, associée à la publication des sources historiques ; 2) la nécessaire question de la modélisation de la « documentation numérique l'histoire des techniques » dans le cadre du développement du Web sémantique.
\end{abstract}

Résumés et mots clés en anglais sont regroupés en fin de volume, accompagnés des mots clés français

Costons omme le montre la thématique de ce numéro de Documents pour l'histoire des techniques, la production scientifique dans le domaine de l'histoire des sciences et des techniques se traduit par un fort développement du recours aux TIC (technologies de l'information et de la communication) : publications de sources en ligne', modélisations d'objets 3D cinématiques², systèmes d'informations géographiques ${ }^{3}$, etc. Partant de travaux de recherches menés à Brest dans les trois domaines suivants : I'histoire des techniques du port-arsenal de Brest, l'éducation', l'ingénierie des environnements

1 Voir par exemple le site « Portail pour l'histoire des sciences et des techniques en France et en Europe » : http:// histsciences.univ-paris 1.fr/index.php? IDcatm=1 \& thema=yes 2 Voir par exemple dans cet ouvrage les contributions de Michel Cotte, Florent Laroche et Stéphane Sire.

3 Voir la contribution de Jean-Louis Kerouanton dans cet ouvrage. 4 La recherche en éducation s'inscrit dans le cadre des activités du groupe ReForEHST : plates-formes.iufm.fr/ehst/rubrique. php3?id_rubrique=3 informatiques pour l'apprentissage humain (EIAH), cette note aura pour objet d'expliciter les problèmes posés aux historiens par l'usage des TIC et dans quelle mesure cette pratique constitue en soi un axe de recherche, ainsi développé dans l'équipe PaHST à l'Université de Brest.

Les recherches en cours en histoire des techniques concernent l'histoire du port-arsenal du XVIIe siècle à l'époque contemporaine au travers des ponts et des grues constituant le paysage portuaire brestois 6 .

5 Voir par exemple : Sylvain Laubé, Serge Garlatti, Jean-Louis Tetchueng, " A scenario model based on anthropology of didactics for Inquiry-Based Science Teaching ", International journal of advanced media and communication, vol. 2, $n^{\circ} 2$, april 2008, pp. 191-208.

6 Sylvain Laubé, " Histoire technique du port-arsenal de Brest : le pont tournant (1861-1944) et le pont à transbordeur (1909-1947) "), dans Philippe Jarnoux, Jean-Lovis Kerouanton, Sylvain Laubé éd., Histoire et techniques du paysage industriel portuaire en France, XVIle-XXe siècles, Rennes, PUR, 2010, à paraître. 
Le point de départ pour cette note est le constat que trois objets technologiques - le pont tournant de Recouvrance (1861-1944), la grue Gervaise et la grue à mâter (1681-1877) - ont fait l'objet de la publication d'images 3D dans différents contextes ${ }^{7}$. Au travers de l'exemple du pont tournant, deux aspects seront discutés : I'historicité de ces images qui implique l'explicitation de la méthode historique à l'œuvre associée à la publication des sources historiques et la nécessaire question de la modélisation de la documentation électronique du point de vue de I'histoire des techniques.

Un exemple de documents numériques(DN)pourl'histoire des techniques : le pont tournant de Recouvrance. Ce pont à deux volées (fig. 1), unique par ses dimensions (plus de 175 mètres de long) et ses mécanismes, était considéré comme un exemple du savoir-faire de l'empire français en matière de travaux publics (et de constructions monumentales) et présenté lors des expositions universelles de 1855 ,

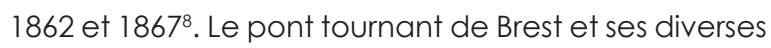
représentations s'insèrent donc dans un cadre général de développement industriel français décidé et démarré en 1852 par Napoléon III. Un bref résumé sera donné ici', d'autant que certains aspects ont été publiés en ligne dans le cadre d'un site internet dédié à la formation des maîtres ${ }^{10}$.

Pour permettre l'ouverture du pont au milieu de la Penfeld, le mouvement de rotation autour des axes des piles est donné par deux cabestans par volée, diamétralement opposés par rapport à l'axe de la pile. Un seul de ces mécanismes suffit pour faire

7 Pour le pont tournant, voir : www.brest3d.fr/pont.php; pour la grue Gervaize : www.wiki-brest.net/index.php/ Image:Capucins_3D_12.jpg; pour la grue à mâter : http:// www.brest3d.fr/maquette-brest2.php

8 La maquette du pont tournant de Brest présentée lors des expositions universelles de Londres (1862) et de Paris (1867) est exposée au Musée national de la Marine, à Brest.

9 Les aspects historiques sont développés notamment dans Sylvain Laubé et Stéphane Sire, "Histoire(s) autour de la maquette du pont tournant de Brest (Musée national de la Marine) "I, Neptunia, n² 247, septembre 2007, pp. 48-53 et Sylvain Laubé et Stéphane Sire, "Histoire du paysage industriel portuaire de Brest au XIXe siècle : l'exemple du premier pont (tournant) de Recouvrance $"$, Actes du Premier congrès francophone d'histoire de la construction, Paris, 19-21 juin 2008 (à paraître, 2010).

10 Voir le site « Ressources en histoire des sciences et techniques pour la formation des maîtres $"$ : /plates-formes.iufm. $\mathrm{fr} /$ ressources-ehst/spip.php?rubrique 18. L'item « Histoire des techniques " a été réalisé en lien avec les AM de Brest ainsi que le service éducatif du Musée national de la Marine.

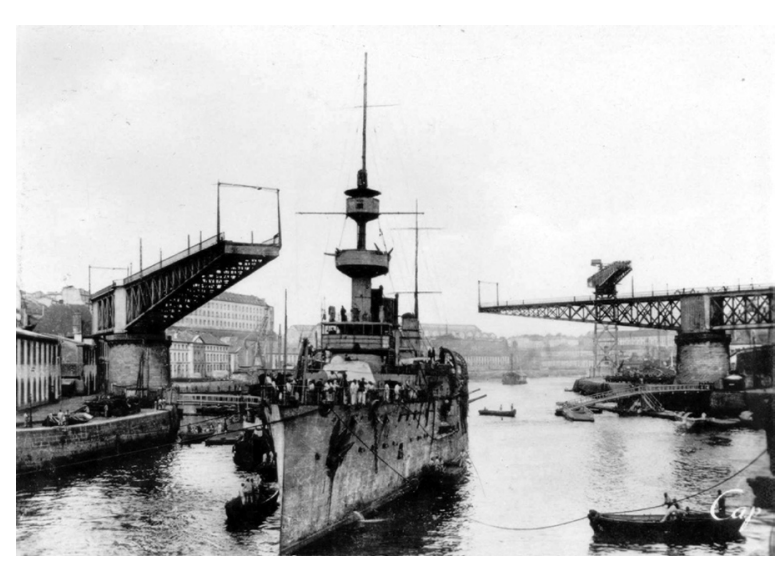

fig. 1 - Le croiseur «Montcalm» sortant du port, le pont tournant ouvert (1937) - Archives municipales de Brest, 3Fi083-067.

tourner une volée lorsque le temps est calme. Les volées pivotent ensuite jusqu'à une position parallèle aux quais. Les recherches ont mis en évidence cinq systèmes mécaniques dont:

- un mécanisme de rotation actionné à bras d'hommes par cabestan et facilité par la présence de rouleaux coniques (sur lesquels est posée la volée) qui minimisent le frottement,

- un mécanisme de verrovillage en bout des deux volées - un mécanisme d'accrochage aux quais (une paire de mâchoires par volée)

- un système de vérins à vis qui prévient la déformation du pont

La figure $2^{11}$ représente un exemple extrait d'une modélisation 3D cinématique réalisée par Stéphane Sire : elle concerne quatre des mécanismes mis en évidence lors des travaux de recherche et plusieurs animations en explicitent la cinématique. Les sources utilisées pour la compréhension des mécanismes comme des contextes historiques d'usages sont issues des divers fonds d'archives disponibles ${ }^{12}$. Les plans de référence à la modélisation sont ceux publiés par M. Aumaître en $1867^{13}$, ingénieur des ponts et chaussées en poste dans le Finistère et chargé de surveiller la conduite des travaux de construction pour l'État.

\footnotetext{
11 S. Laubé, S. Sire, 2007, op. cit., note 9.

12 A titre d'exemple, voir : les fonds (2S 70, 2S 71 \& 2S 72), AD Finistère ; Nicolas Cadiat et Alphonse Oudry, Projet de pont entre Brest et Recouvrance, Fonds anciens de l'école des Ponts et Chaussées, cote 4.4735/C261, 1854 ; le fond iconographique numérisé des Archives municipales de Brest en ligne : http://archives.mairie-brest.fr/4DCGI/Web_DF/ILUMP4763

13 M. Aumaître, « Note $n^{\circ} 167$ relative au pont tournant construit sur la Penfeld pour la traverse de la route impériale $\mathrm{n}^{\circ} 12$ dans la ville de Brest " Annales des ponts et chaussées, 4ème série, tome 14, 1867, pp. 265-276. On trouve la revue numérisée ici : http://gallica.bnf.fr/ark:/12148/bpt6k408508k.r=.langfr
} 

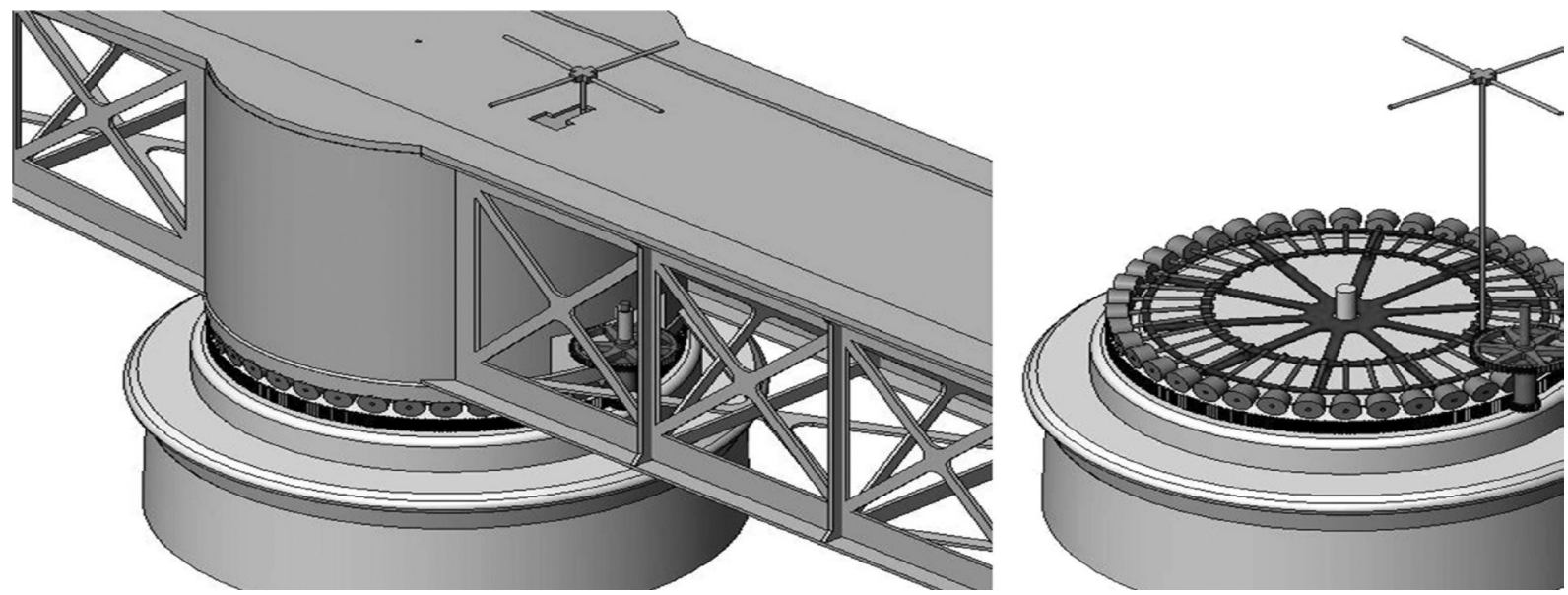

fig. 2. (ci-dessus) - Le mécanisme de rotation du pont tournant (1867) (auteur: S. Sire, [Laubé \& Sire, 2007]) À gauche, en rouge, le cabestan déployé et mis en action par les agents de la direction du port. La volée est posée sur les rouleaux et on aperçoit un des engrenages en bleu. À droite, la volée a été enlevée. Le cabestan (en rouge) entraîne un petit pignon qui engrène sur le pignon bleu qui engrène à son tour sur la crémaillère afin d'opérer la rotation de la volée.

fig. 3 (droite) - Le mécanisme de rotation du pont tournant (auteur : Roger Yven, http://www.bresł3d.fr/pont.php).

La figure 3 provient d'un site ${ }^{14}$ qui présente plusieurs vues du pont ainsi qu'un montage vidéo comportant cartes postales et plans du mécanisme de rotation, les sources étant non référencées. Une animation 3D montre le mécanisme ainsi qu'une vue du pont en rotation. Une première lecture de ces deux images (sans connaissances a priori du contexte de production et sans analyse de la situation de communication) indique une très grande similitude avec le constat d'une plus grande qualité esthétique pour la figure 3, dû à la colorisation et aux textures qui génèrent un effet de réalité.

\section{De l'historicité du document numérique à la question du genre du DN pour l'histoire des techniques}

La question de l'historicité ${ }^{15}$ de ces deux images se pose alors. La citation des sources en constitue la première étape. La figure 2 est insérée dans un discours historique et un corpus de référence constitués par la recherche (très brièvement décrits décrits au-dessus).

Si la page internet où est publiée la figure 3 (avec son animation) présente une iconographie

14 Voir Roger Yven, www.bresł3d.fr/pont.php, site dédié, pour partie, à la « reconstitution historique ».

15 Au sens de ce qui peut être attesté de manière factuelle par la « critique des sources».

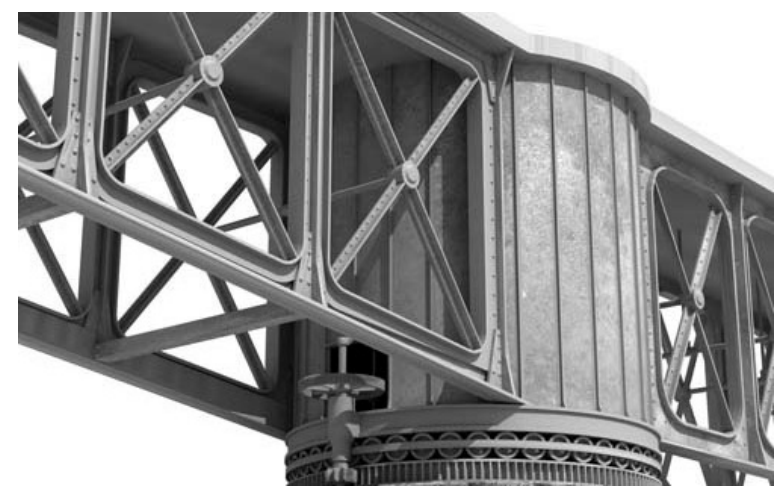

historique, les références des sources sont absentes. On peut aisément les retrouver : d'une part, sur le site des Archives municipales de Brest pour les cartes postales, d'autre part, sur le site "Digital Bridges " pour les deux planches ${ }^{16}$.

L'image 3D est un modèle qui résulte de l'analyse critique des sources historiques. Deux points extraits de nos exemples permettent de le montrer. Chacun des modèles montrent un seul mécanisme pour la rotation d'une volée du pont. Les sources indiquent que deux mécanismes identiques étaient situés symétriquement par rapport à l'axe de rotation de chaque volée. Selon les conditions (climatiques par exemple), on opérait à partir d'un ou deux mécanismes avec des équipes jusqu'à huit hommes par cabestan. Le modèle 3D de la figure 2 ne constitue donc pas une volonté de reproduire au plus près la réalité historique telle qu'on la connaît, mais d'expliciter un mécanisme du point de vue de l'histoire des techniques (qui constitue de fait le contexte de production de cette image). De même, les plans issus des sources historiques ne présentent qu'un seul mécanisme, par souci de faciliter la compréhension du lecteur et éviter une redondance néfaste.

Nous faisons ici l'hypothèse que le modèle 3D de

16 http://digital.lib.lehigh.edu/cdm4/bridges_viewer.php?D MTHUMB $=\& C I S O P T R=3124 \& p t r=3168$ 
la figure 3 résulte de l'interprétation directe de la seule source citée qui ne montre qu'un seul mécanisme pour la rotation, sans analyse critique. Si elle présente certaines qualités pour expliciter le mécanisme à l'œuvre, elle ne constitue pas une image à caractère historique. Cette hypothèse paraît confirmée par le fait, par exemple, que les croix de Saint-André manquent dans la structure métallique sous la volée. Par ailleurs, la page d'accueil' ${ }^{17}$ nous informe sur le contexte de production : publié par une petite entreprise d'images de synthèse et de graphisme, il s'agit de démontrer ici de compétences en imagerie 3D au travers d'exemples particulièrement frappants et esthétiquement très séduisants. L'auteur indique cependant dans ces compétences: «reconstitutions historiques $11^{18}$.

Une première conclusion à partir de ces deux exemples réside dans la difficulté à déterminer a priori dans quelle mesure un modèle $3 \mathrm{D}$ est historique et donc la nécessité de définir de manière plus générale le genre « Document Numérique (DN) » pour l'histoire des techniques ${ }^{19}$.

Michael Shepherd et Livia Polanyi20, dont le travail est cité par loannis Kanellos ${ }^{21}$, proposent de caractériser le genre propre au DN par trois éléments " constitutifs»:

- Le contenu (information ...), organisé suivant une structure matérielle (disposition, mise en page, etc.), souvent suffisante lors d'une première et rapide lecture pour deviner le genre, et logique (titre, auteur, date, résumé etc.), qui apporte de l'information sur l'organisation intellectuelle du document.

- Le contenant (support, médium), qui détermine le mode d'accès, d'appropriation ou de "lisibilité » de l'information (par l'homme ou la machine).

- Le contexte de production, qui retrace l'intention de publication, dans un cadre, une fonction ou une activité donnée. Jouant un rôle essentiel dans

\footnotetext{
17 http://www.brest3d.fr/

18 Ce qui nous paraît aller au-delà de la réalité.

19 Ou encore le cybergenre du « document pour l'histoire des techniques $॥$.

20 Michael Shepherd, Livia Polanyi, " Genre in digital documents "1, 33 rd Hawaii international conference on system sciences, volume 3, 2000, «pp. 3010 », www.computer.org/portal/ web/csdl/abs/proceedings/hicss/2000/0493/03/0493toc.htm.

21 Voir loannis Kanellos, Thomas Le Bras, Frédéric Miras, loana Suciu, "Le concept de genre comme point de départ pour une modélisation sémantique du document électronique ", Actes du colloque international sur le document électronique (CIDE'05), Beyrouth, Liban, avril 2005, pp. 201-216.
}

le processus de lecture du document, le contexte peut être en partie reflété tant dans le contenu que dans le contenant.

Si nous appliquons ce schéma du genre à nos deux exemples (figure 2 et 3 ), les contextes de production sont pour nous clairement identifiés : I'un est celui de la recherche en histoire des techniques, l'autre du domaine de l'activité commerciale dans le domaine de l'imagerie 3D. On constate par contre que les contenants (les images de synthèse associées à leur cortège d'animations cinématiques) sont très proches et que les contenus les plus lisibles (expliciter le mécanisme de rotation) sont eux aussi très semblables. Les contenants comme les contenus en tant que tels ne permettent pas de décider aisément du contexte de production et donc du genre du DN (historique ou non). Seules les informations publiées dansl'environnement du DNidentifientet déterminent par conséquent le contexte de production, par suite le genre.

Par ailleurs, la genèse du DN peut être décrite par les processus suivants ${ }^{22}$ :

- La réplication (reproduction) des genres par rapport aux genres préexistant, qui se retrouvent dans d'autres médias.

- L'émergence de nouveaux genres qui n'ont pas d'équivalent sur support analogique ou dans un autre média.

Dans notre exemple, la modélisation de l'imagerie 3D (cinématique ou non) pour l'histoire des techniques ${ }^{23} s^{\prime}$ inscrit dans le second processus (l'émergence d'un nouveau genre de DN), mais on a vu plus haut une possible difficulté à définir le contexte de production, pour le lecteur «non initié » ou non, à partir du seul contenu et du contenant.

22 Michael Shepherd et Carolyn Watters, "Identifying web genre: hitting a moving target", WWW 2004 Conference workshop "Measuring web search effectivness: the user perspective", New-York, 18 mai 2004, www.cs.dal.ca/ watters/www2004WorkShop/pdfs/4.pdf, 23 Voir les travaux de Laroche et al dont on trouvera ici la bibliographie : http://florent.laroche.free.fr/blog/index. php? Liste-des-publications-scientifiques. 
La communauté de pratique ${ }^{24}$ de référence (ici, des historiens des techniques) définit le contexte de production tout comme l'organisation logique des connaissances etc'estalors le processus de réplication qui rendra le DN plus facilement acceptable et utilisable par la communauté de pratique ${ }^{25}$.

Le site internet " Plan de Rome, restituer la Rome

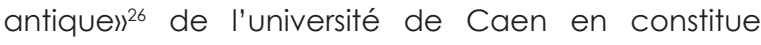
un exemple intéressant à analyser. En effet, si l'on considère le site en tant que tel comme un DN numérique (incluant de l'imagerie 3D virtuelle interactive et cinématique), on se trouve dans une situation de réplication d'un genre déjà connu en histoire : celui de la restitution. Le point de départ de cette recherche est la présence à Caen du plan relief de Paul Bigot, appelé le «Plan de Rome ${ }^{27}$, réalisé à partir du début du siècle dernier ${ }^{28}$.

L'explicitation de ce projet numérique montre bien son insertion dans un genre déjà reconnu par les historiens et les archéologues qui comporte cependant une part en émergence : la modélisation qui nécessite une articulation entre infographistes et chercheurs (qui indique qu'une nouvelle communauté de pratique est en cours à partir de celles des historiens et des informaticiens).

Nous retiendrons de ce paragraphe l'idée que le modèle 3D virtuel en lui-même, non contextualisé, est a priori a-historique. C'est non seulement l'explicitation de la méthode de modélisation au regard de celle de l'histoire des techniques qui lui permet de s'inscrire dans le genre « DN pour l'histoire des techniques » mais aussi la publication des sources

24 Les communautés de pratique se définissent selon trois dimensions : les frontières de leur domaine d'application, leur existence sociale en tant que communauté et les outils, le langage, les histoires et documents que les membres de cette communauté partagent et s'échangent. C'est un groupe qui interagit, apprend ensemble, construit des relations et à travers cela développe un sentiment d'appartenance et de mutuel engagement. Voir Etienne Wenger, Communities of practice: learning, meaning, and identity, Cambridge University Press, 1998 ou encore son site : http://www.ewenger.com/theory/ 25 C'est le cas par exemple du « Portail pour l'histoire des sciences et des techniques en France et en Europe 11 , voir note 1 ; ou encore de la publication en ligne de sources numérisées pour I'histoire des techniques, voir le site du Cnum : cnum.cnam.fr/ 26 www.unicaen.fr/services/cireve/rome/index.php

27 Philippe Fleury, Sophie Madeleine, "Problématique d'une restitution globale de la Rome antique. Une visite interactive avec accès dynamique aux sources anciennes $"$, colloque international Virtual retrospect, Bordeaux, 15-16 novembre 2007 ; http://www.unicaen.fr/services/cireve/rome/pdf/article.pdf $28 \mathrm{http}: / /$ www.unicaen.fr/services/cireve/rome/pdr_maquette. php?fichier=bigot utilisées pour construire le modèle (et qui autorise la critique des sources pour le chercheur/utilisateur). Ce point amène à penser le DN inséré nécessairement dans un environnement informatique pour l'histoire des techniques (EIHT) dont l'organisation dépendra fortement du contexte d'utilisation.

Perspectives pour la recherche à Brest : du genre du document numérique pour l'histoire des techniques à la question de sa modélisation informatique pour le web sémantique Le développement du web sémantique dont l'objectif est de fournir un cadre commun qui permet aux données en ligne d'être partagées et réutilisées pour diverses applications ${ }^{29}$ génère des questions de recherche de nature pluridisciplinaire car il s'agit de modéliser d'un point de vue informatique les DN en fonction des communautés de pratiques qui les utilisent. Appliqué au cas d'un EIHT, cela nécessite de modéliser le genre du « DN en histoire des techniques ॥ tel qu' on a pu le définir dans cette note. Par ailleurs, on a besoin d'ontologies ${ }^{30}$ qui permettent de décrire le domaine des connaissances pour pouvoir réutiliser les DN dans des environnements dédiés à la recherche mais aussi à l'enseignement.

Un travail préliminaire d'élaboration d'une ontologie définissant les sources historiques concernant les grues et les ponts de l'arsenal de Brest est en cours en s'appuyant notamment sur les travaux de Kanellos ${ }^{31}$, et en collaboration avec Serge Garlatti, chercheur au département d'informatique de Telecom-Bretagne.

\|l s'inscrit en lien avec les deux projets européens dans le domaine de la recherche sur les EIAH où l'équipe PaHST est chargée de deux missions. II s'agit d'une part d'élaborer des lignes de conduite (" guide-lines ») pour analyser et construire des EIAH basés sur la démarche d'investigation en sciences et utilisant des ressources en histoire des sciences et techniques ${ }^{32}$. D'autre part, à partir de ce " guide-line », l'enjeu est de construire et d'évaluer un EIAH pour la formation des maîtres concernant les méthodes innovantes pour enseigner en utilisant des ressources en histoire des sciences et techniques.

29 Voir par exemple : http://www.w3.org/2001/sw/

30 Une ontologie constitue en soi un modèle de données représentatif d'un ensemble de concepts dans un domaine, ainsi que les relations entre ces concepts. Elle est employée pour raisonner à propos des objets du domaine concerné. Voir par exemple : http://fr.wikipedia.org/wiki/Ontologie_(informatique.

31 Voir lonnis Kanellos, « Lecture(s) et genre(s) du document numérique " Xle colloque international sur le document électronique : Interactions \& usages autour du document numérique (CIDE 11), Rouen, Trupin éd., 2008, pp. 202-210. 32 « Mind the Gap », contrat FP7 n² 217725. 